\title{
PLK-1 inhibition improves abiraterone efficacy
}

New data show that activation of pololike kinase 1 (PLK-1) through oxidative stress has a central role in upregulation of pathways that promote and sustain the development of castration-resistant prostate cancer (CRPC), and that inhibition of this serine-threonine kinase potentiates the therapeutic effect of abiraterone in CRPC xenografts.

\section{The combination ... is likely to have a huge effect on treatment of CRPC 77}

PLK-1 is a cell cycle regulator that has been linked to neoplastic transformation in many human tumours, including prostate cancer. Indeed, the PLK-1 inhibitor BI2536 is being investigated in phase II clinical trials in patients with lung cancer and leukaemia. Interestingly, previous microarray analyses found that both PLK-1 and lipid metabolism pathways were highly upregulated in prostate cancer xenografts. "It has been well established that oxidative stress, the PI3K-AKT-mTOR pathway, androgen receptor signalling and cholesterol metabolism are all involved in prostate cancer progression, but how these mechanisms are coordinated is unclear," explains Xiaoqi Liu, senior investigator of the study published in Cancer Research.

Using a panel of prostate cancer cell lines, the researchers discovered that oxidative stress increased levels of PLK-1 and activated the PI3K-AKT-mTOR pathway and androgen receptor expression in a PLK-1-dependent manner in vitro.

Next, Liu and his team, who had previously found increased levels of cholesterol esters (precursors of testosterone) in high-grade prostate cancer, demonstrated that oxidative stress increased and that inhibition of PLK-1 by BI2536 reduced intracellular cholesterol ester levels. In addition, they found evidence of positive feedback loops between PLK-1-dependent upregulation of androgen receptor signalling, the PI3KAKT-mTOR pathway and cholesterol ester synthesis, as well as between androgen receptor signalling and $P L K 1$ expression.

Importantly, combination of BI2536 with enzalutamide in cell lines or abiraterone in CRPC xenografts in mice was more effective than any of the drugs alone. In vivo, the combination therapy curbed tumour growth and elevation of serum PSA levels, in contrast to the single agents, and significantly reduced testosterone levels and considerably decreased the number of intracellular lipid droplets.

"As BI2536 is already in clinical trials, safety concerns have been addressed," highlights Liu. "The combination of BI2536 with androgen signalling inhibitors is likely to have a huge effect on treatment of CRPC."

Clemens Thoma

Original article Zhang, Z. et al. Plk1 inhibition enhances the efficacy of androgen signaling blockade in castrationresistant prostate cancer. Cancer Res. doi:10.1158/ 0008-5472.CAN-14-1916 